\title{
The adaptor protein $\mathrm{p} 40^{p h o x}$ as a positive regulator of the superoxide-producing phagocyte oxidase
}

Futoshi Kuribayashi, Hiroyuki Nunoi, Kaori Wakamatsu, Shohko Tsunawaki, Kazuki Sato, Takashi Ito \& Hideki Sumimoto

Correction to: The EMBO Journal (2002) 21: 6312-6320. DOI 10.1093/emboj/cdf642 | Published online 1 December 2002

\section{Authors' statement}

We have found that in Figure 2C, one panel is mistakenly a duplication of the adjacent panel. The two images of "Blot: anti-p47(phox)" and "Blot: anti-p67(phox)" are the same, albeit with distinct contrast. According to our inspection of the original data sets, this was due to a mistake during the assembly of the panels. We show here the amended Figure $2 \mathrm{C}$, with the correct image for the panel of p67 (phox) expression.

The annotated source data for this panel and the anti-p47(phox) panel is provided. The change does not affect the original conclusions presented.

Original data for the corrected figure: detection of $\mathrm{p} 47^{\text {phox }}$ by immunoblot

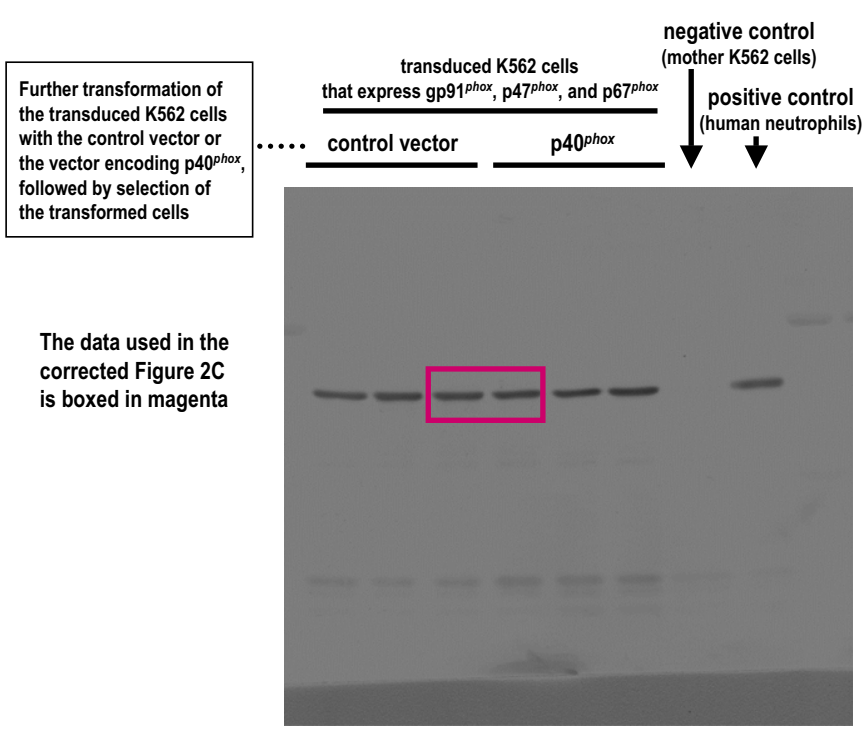

Source data for Figure 2C (anti-p47(phox)).
C

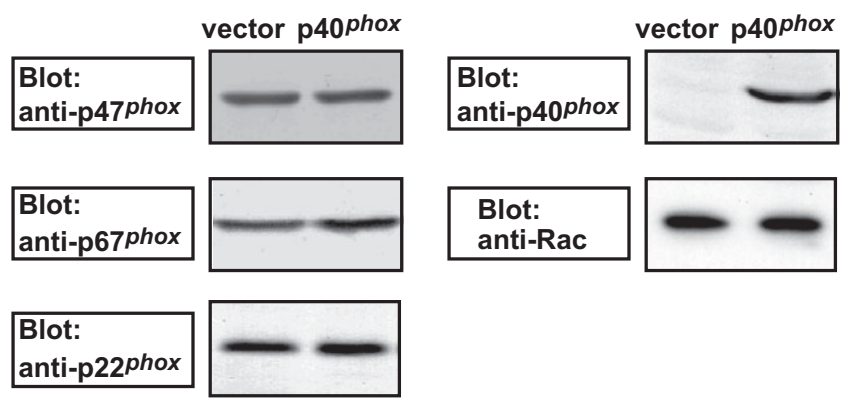

Figure 2C.

Original data for the corrected figure: detection of $\mathrm{p} 67^{\text {phox }}$ by immunoblot

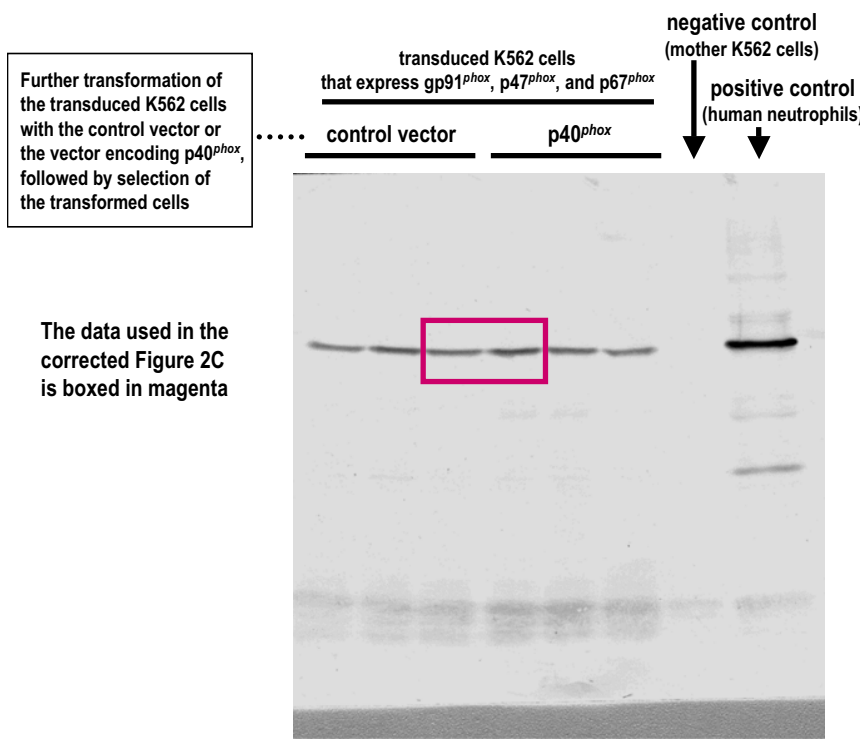

Source data for Figure 2C (anti-p67(phox)). 


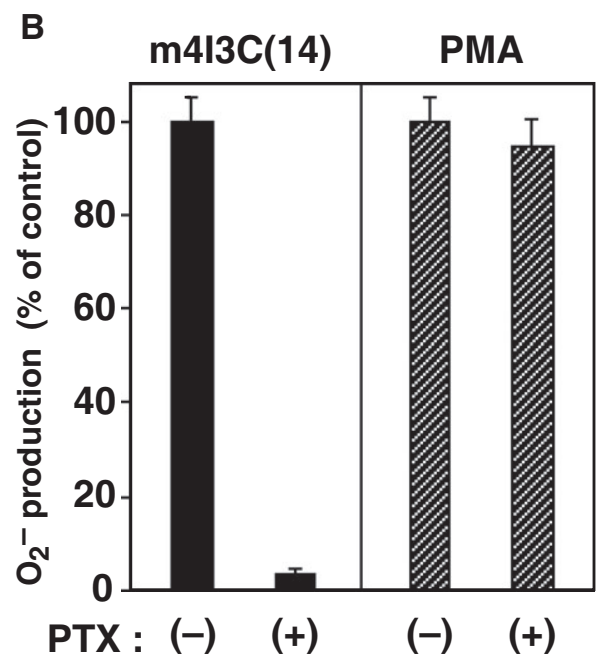

Figure 8B.
In addition, we have also discovered errors in the labelling of Fig $8 \mathrm{~B}$; each “ $(+)$ ” should have read " $(-)$ ”, whereas each " $(-)$ ” should have read " $(+)$ ". These changes are consistent with the description of the data in the Results section, and thus do not affect the original conclusions presented.

All the authors have evaluated the mistakes and agree with the revised figures as presented. The authors apologize for any inconvenience caused and the delay in correcting them. 